\title{
Positionspapier der DMV zur Verwendung bibliometrischer Daten
}

\begin{abstract}
Bibliometrische Daten werden heute zunehmend in der Evaluation von Forschungsergebnissen benutzt. Diese Anwendungen reichen von der (indirekten) Verwendung bei der Peer-Evaluation von Drittmittelanträgen über die Beurteilung von Bewerbungen in Berufungskommissionen oder Anträgen für Forschungszulagen bis hin zur systematischen Erhebung von forschungsorientierten Kennzahlen von Institutionen. Mit diesem Dokument will die DMV ihren Mitgliedern eine Diskussionsgrundlage zur Verwendung bibliometrischer

Daten im Zusammenhang mit der Evaluation von Personen und Institutionen im Fachgebiet Mathematik zur Verfügung stellen, insbesondere auch im Vergleich zu anderen Fächern. Am Ende des Texts befindet sich ein Glossar, in dem die wichtigsten Begriffe kurz erläutert werden.
\end{abstract}

\section{Zusammenfassung}

Die DMV appelliert an alle Beteiligten, sich der Problematik rein numerischer Daten bewusst zu sein und eine faire, dem Fach angemessene Bewertung sicherzustellen. Insbesondere bei Entscheidungen, welche die Karrierechancen von Wissenschaftlerinnen und Wissenschaftlern beeinflussen, sollten bibliometrische Daten nur in Verbindung mit einem Peer Review Verfahren eingesetzt werden.

Im Folgenden werden die wichtigsten Prinzipien und fachspezifischen Gegebenheiten zusammengefasst:

- Qualität der Daten

- Die verwendete Datenbasis muss transparent offen gelegt werden.

- Die Daten müssen gut gepflegt und der untersuchten Fragestellung angemessen sein.

- Automatisch erstellte Autoren- und Institutsidentifikationen sind grundsätzlich fehleranfällig.

- Spezifika der Mathematik

- Werte wie $h$-Indizes oder Impaktfaktoren sind in anderen Wissenschaften (z. B. Life Sciences) in der Regel deutlich höher. Dies ist per se kein Qualitätsmerkmal.

- Die Halbwertszeit mathematischer Arbeiten liegt weit über der in anderen Fachgebieten.

- Der Zeitraum zwischen Erstellung und Verteilung eines Preprints (etwa über arXiv) und der endgültigen Veröffentlichung kann auf Grund der genauen inhaltlichen Prüfung oft Jahre betragen.

- Die Mathematik kennt in der Regel nicht den Begriff des Erstautors. Die Namen der Autoren werden alphabetisch angeordnet.

- Problematik von Indikatoren

- Indikatoren können durch das Verhalten von Autoren bewusst beeinflusst werden. In einigen Fällen werden Indikatoren gezielt manipuliert.

- Die Berechnungsmethodik einiger Faktoren, etwa des Impaktfaktors von Zeitschriften, ist den Spezifika der Mathematik nicht angepasst.

- In vielen Fällen ist die Datengrundlage für die Berechnung von Indikatoren unklar. Verlässliche Informationen zu (abschließend veröffentlichten) mathematischen
Publikationen liefern vor allem die Datenbanken zbMATH und MathSciNet.

- Ranking von Zeitschriften

- Auf Grund der Erfahrungen anderer mathematischer Gesellschaften verzichtet die DMV bewusst auf ein Ranking von Zeitschriften.

- Die Einschätzung verschiedener Zeitschriften ist abhängig von den betrachteten Gebieten und unterliegt einem zeitlichen Wandel.

- Grundsätzlich gilt, dass jede Veröffentlichung nach ihrem wissenschaftlichen Inhalt und nicht nach dem $\mathrm{Pu}$ blikationsort zu bewerten ist.

$\mathrm{Zu}$ beachten ist auch, dass eine wissenschaftliche Leistung heute zunehmend neben der eigentlichen Publikation auch die Erstellung mathematischer Software oder die Erzeugung von Forschungsdaten beinhalten kann.

\section{Detaillierte Darstellung}

Die oben angesprochenen Punkte umfassen viele Detailaspekte, die hier ausführlicher diskutiert werden sollen.

\section{Qualität der Daten}

Grundlage jeglicher Evaluation müssen gesicherte und qualitativ hochwertige Daten sein. Insbesondere sind zu beachten:

- Es ist zu überprüfen, in wieweit die Daten im Hinblick auf die betrachtete Fragestellung vollständig sind oder ob die Datenbasis einer impliziten Vorauswahl unterliegt. Ein Beispiel ist das Web of Science, welches viele mathematische Zeitschriften nicht indiziert. So wurden beispielsweise für das Jahr 2017 laut Journal Citation Reports in der Kategorie Mathematics lediglich 310 Journals ausgewertet, vgl. [28]. Im Vergleich dazu indexieren etwa MathSciNet und zbMATH aktuell ca. 18 oo Zeitschriften, inklusive Buchserien sind es bei zbMATH sogar mehr als 3.ooo Periodika [32], [34].

- Es ist zu prüfen, welche Qualitätskriterien der Datenauswahl zugrunde liegen. Gibt es aktive Kontrollmechanis- 
men, welche etwa die Relevanz der Daten oder die gute wissenschaftliche Praxis der zitierten Journale regelmäBig überprüfen? Zitationsfarmen können die Ergebnisse schnell komplett verfälschen.

- Autorendisambiguierung kann ein Problem sein, insbesondere bei häufig auftretenden Namen oder bei Transkriptionen aus anderen Alphabeten.

- Eine automatische Zuordnung von Veröffentlichungen zu Institutionen kann durch die Verwendung unterschiedlicher Institutionsbezeichnungen (etwa deutsch/englisch) und die üblichen langen Publikationszeiten (in denen Autoren die Institution wechseln können) erschwert werden.

- Datenbanken wie zbMATH und MathSciNet (siehe Glossar) sind eine geeignete Grundlage, da sie im Wesentlichen vollständige, gut gepflegte und mathematisch relevante Daten enthalten. Allerdings ist die zeitliche Verzögerung, die sich durch die oft langen Publikationsabläufe (siehe unten) ergeben kann, zu beachten.

\section{Spezifika der Mathematik}

Bibliometrische Daten werden oft benutzt, um einen Vergleich zwischen verschiedenen Fächern oder Fakultäten herzustellen. In diesem Zusammenhang ist es wichtig, für die Mathematik spezifische Sachverhalte zu berücksichtigen. Um unangemessene Verzerrungen zu vermeiden, sind insbesondere folgende Punkte zu beachten:

- $h$-Indizes oder Impaktfaktoren sind in anderen Bereichen wie etwa Physik, Chemie und insbesondere Life Sciences deutlich höher. Gründe liegen in der Größe der Fachcommunity, dem unterschiedlichen Publikationsverhalten und der typischen Anzahl Autoren pro Publikation. Selbst innerhalb der Mathematik kann es je nach fachlicher Ausrichtung beziehungsweise der Nähe zu Anwendungsfächern erhebliche Unterschiede geben, [9].

- Es ist ein Charakteristikum der Mathematik, dass viele Veröffentlichungen teilweise erst nach geraumer Zeit in der Literatur zitiert werden. Gleichzeitig liegt die Halbwertzeit mathematischer Veröffentlichungen deutlich höher als in anderen Fächern, [5-7]. Es gibt zahlreiche Publikationen, die lange Zeit nicht oder nur selten „nachgefragt" sind und mit einer neuen Forschungsfrage plötzlich in den Fokus des Interesses treten. Dabei kann es bei der Rezeption zu maßgeblichen zeitlichen Abständen kommen. Beispiele von bis zu 100 Jahren sind keine Seltenheit, wie bei einer Dissertation aus dem Jahre 1907 ersichtlich wird, [18], vgl. [3], oder einer Serie von Arbeiten aus dem Jahre 1913, [12-14], vgl. [16]. Diese Effekte wiederum haben Auswirkungen auf die (zeitliche) Entwicklung von Impaktfaktoren oder $h$-Indizes.

- Der Veröffentlichungsprozess in der Mathematik dauert im Vergleich zu anderen Fächern erheblich länger, ein bis zwei Jahre zwischen Einreichung und Erscheinen sind durchaus normal, noch ausgedehntere Zeitspannen sind möglich. Dies liegt an den aufwändigen Begutachtungsverfahren, welche nicht nur eine Aussage über die Relevanz eines erzielten Ergebnisses machen, sondern auch eine inhaltliche Prüfung einzelner Beweisschritte umfasst. Diese zeitliche Verzögerung kann insbesondere bei der Bewertung jüngerer Wissenschaftler zu Problemen führen, [17].

- Die Mathematik kennt in aller Regel nur die alphabetische Anordnung von Autorinnen und Autoren. Es gibt keinen Begriff der Erstautorenschaft, eventuelle Rückschlüsse aus der Autorenreihung sind unzulässig. Ausnahmen hiervon können entstehen bei Übersetzungen der Autorennamen aus anderen Alphabeten oder bei stark interdisziplinären Arbeiten, die den Gepflogenheiten anderer Fächer folgen. (Es gibt Einzelfälle, z. B. an einigen chinesischen Universitäten, wo bibliometrische Daten für alle Fächer gleich behandelt werden. Hier kann Erstautorenschaft eine Rolle spielen.)

○ Konferenz- und Sammelbände beinhalten oft wichtige Beiträge für die mathematische Literatur, vor allem auf dem Gebiet der Übersichtsartikel. Dennoch ist festzuhalten, dass die Konferenzbeiträge nicht denselben Stellenwert besitzen, den sie teilweise in den Ingenieurwissenschaften und der Informatik haben; und Grund hierfür ist, dass mathematische Tagungen eher als Einzelevents und nicht im Rahmen langjährig etablierter Tagungsserien mit vielen Teilnehmern organisiert werden.

Konkret hatte zum Beispiel Peter Scholze zum Zeitpunkt seiner Berufung 2012 in Bonn noch überhaupt keine Zitierungen seiner Arbeiten. Eine Einbeziehung bibliometrischer Indikatoren in die Berufungsentscheidung hätte möglicherweise den Verlust eines künftigen Fields-Medaillenträgers für die deutsche Universitätslandschaft bedeutet.

Es ist Teil der mathematischen Publikationskultur, dass es als schlechter Stil gilt, Mathematik in „least publishable units" zu veröffentlichen: Gute Arbeiten sollen auch in vielen Jahren noch relevant und gut lesbar sein, weswegen erwartet wird, dass die Autoren ihr Werk mit entsprechender Sorgfalt verfassen und ggf. „reifen lassen“.

\section{Diskussion von Indikatoren}

Es gibt verschiedene Kennzahlen, deren Besonderheiten und grundsätzliche Probleme zu beachten sind. Bei der Evaluation von Einzelpersonen ist insbesondere zu beachten:

Zitationszahlen: Der einfachste Ansatz besteht in einer reinen Zählung von Zitaten. Es besteht zwar Übereinstimmung darin, dass hohe Zitationszahlen in der Regel ein Ausweis für wissenschaftliche Sichtbarkeit sind, dennoch ergeben sich offensichtliche Probleme. Die Zahl der Zitate hängt (stark) von der gewählten Datenbasis (Preprints oder veröffentlichte Arbeiten, Datenbank) ab. Nicht immer werden die Arbeiten zitiert, in denen der entscheidende Durchbruch erzielt wurde; manchmal werden stattdessen relativ einfache Verallgemeinerungen, Lehrbücher oder Übersichtsartikel zitiert. Es gibt zudem verschiedene Gründe, weshalb Arbeiten zitiert werden, nicht alle Zitationen sind positiv zu bewerten. In Extremfällen werden Zitationszahlen künstlich aufgebläht.

$h$-Index: Der $h$-Index (Hirsch-Index) eines Wissenschaftlers ist die größte Zahl $h$, so dass ein Autor bzw. eine Autorin $h$ Arbeiten hat, die mindestens $h$-mal zitiert werden, siehe [15]. Der hier verfolgte Ansatz ist, die reine Zählung von Zitationen zu relativieren. Der $h$-Index 
ist inzwischen ein weithin populäres Maß, insbesondere da (zumeist ohne Auswahl eines Auswertungszeitraums) die Anzahl von Artikeln und ihren Zitationen auf eine einzige Zahl abgebildet wird. Wegen geringerer Beeinflussbarkeit durch wenige vielzitierte Arbeiten oder kleine Fehler in Zitationszuordnungen wird er oft als robuster und weniger manipulationsanfällig wahrgenommen. Der $h$-Index hat jedoch verschiedene strukturelle Unzulänglichkeiten: Unter anderem benachteiligt er junge Wissenschaftler(innen), ist in hohem Maße gebietsabhängig, kappt die Information von singulären Durchbrüchen, und setzt Anreize zur Veröffentlichung von „least publishable units“ zur simultanen Maximierung von Publikations- und Zitationszahl. Zudem wird der $h$-Index typischerweise ohne Differenzierung nach Gebieten erstellt, was in der Mathematik wegen der unterschiedlichen Zitationszahlen oft $\mathrm{zu}$ vergleichsweise niedrigen Zahlen führt. Die $h$-Indizes, die etwa von Google Scholar, Scopus oder Web of Science ausgewiesen werden, ergeben numerisch sehr unterschiedliche Werte, [11].

Modifizierte h-Indizes: Inzwischen wurden mehrere Modifikationen des ursprünglichen h-Index vorgeschlagen, welche beispielsweise nur Publikationen der letzten Jahre berücksichtigen oder verschiedene Gewichtungen vornehmen. Die meisten grundsätzlichen Probleme bleiben jedoch bestehen.

Altmetrics: In jüngerer Zeit gab es verschiedene Versuche, mit webbasierten Metriken die Reichweite z. B. in sozialen Netzwerken, Medien etc. zu messen. Offenbar sind diese noch leichter manipulierbar (z. B. TwitterBots); darüber hinaus führen sie zur Dominanz öffentlichkeitswirksamer, leicht zugänglicher oder kontroverser Themen. Für die Mathematik konnte bisher keine Aussagekraft nachgewiesen werden.

Für die Evaluation von Zeitschriften gilt:

Impaktfaktor (von Zeitschriften): Nach klassischer Definition misst dieser Faktor, wie oft Arbeiten einer Zeitschrift in den vergangenen zwei Jahren durchschnittlich in anderen Veröffentlichungen zitiert werden ein Zeitraum, der für die Mathematik (s.o.) viel zu kurz ist. Alternativ gibt es auch Impaktfaktoren, welche einen Zeitraum von fünf Jahren berücksichtigen. Grundsätzlich gilt, dass es eine gefühlte Korrelation zwischen den bekannten Impaktfaktoren vieler Journale und der intuitiven Einordnung dieser Zeitschriften gibt. Allerdings lässt der Impaktfaktor einer Zeitschrift keinerlei gesicherte Rückschlüsse auf die wissenschaftliche Qualität eines einzelnen Artikels in dieser Zeitschrift zu. Ferner variiert er stark mit dem Arbeitsgebiet, der Größe der angesprochenen Community und dem Profil der Zeitschrift. Auch hier ist es wichtig, die Datenmenge zu spezifizieren, welche die Berechnungsgrundlage für die Impaktfaktoren ist.

h-Index für Journale: Grundsätzlich gibt es auch verschiedene Formen von $h$-Indizes und vergleichbare Werte für Zeitschriften. Auf diese treffen die gleichen Bemerkungen wie oben zu.
Eigenfaktor: Beim Eigenfaktor handelt es sich um ein Maß, das auf der Zentralität in Zitationsnetzwerken beruht. Dies führt zu einer unterschiedlichen Gewichtung von Zitationen. Während dieser Zugang zunächst für Zeitschriften entwickelt wurde, gibt es inzwischen auch Variationen für einzelne Autoren. Es ist als Maß nicht etabliert und von der Verwendung wird abgeraten.

Besonders zuverlässige Datenquellen für bibliometrische Daten in der Mathematik sind die Datenbanken zbMATH und MathSciNet. Hierbei handelt es sich um die Standardreferenzdatenbanken in der Mathematik, die sowohl bibliographische Daten als auch Besprechungen der betreffenden Artikel, Werke etc., sogenannte Referate („Reviews“), enthalten. Insgesamt (einschließlich der Daten aus dem „Jahrbuch über die Fortschritte der Mathematik“) wird hierdurch ein Zeitraum bis in das Jahr 1868 zurück abgedeckt. Bei beiden Datenbanken kann man darüber hinaus umfangreiche Informationen über einzelne Journale (Zahl der Publikationen, Zitate) abfragen. MathSciNet berechnet daraus einen 5-Jahres-Impaktfaktor, zbMATH bietet Profile mit detaillierten Zusatzinformationen (Gebiete, Autoren, Quellen) für Publikationen und Zitationen an und bietet mit swMATH auch einen Nachweis für die Verwendung von Softwarepaketen in der mathematischen Literatur an.

\section{Ranking von Zeitschriften}

In vielen Diskussionen in der mathematischen Gemeinschaft wird von einer impliziten Übereinkunft darüber ausgegangen, welche Zeitschriften zu den „Topjournalen“ in der Mathematik gehören. Dies ist kritisch zu reflektieren. Zum einen sind auch hier Gebietsspezifika zu berücksichtigen. Zum anderen können sich die Qualität und das Profil von Zeitschriften zeitlich verändern (und tun dies auch). Gerade im Zusammenhang mit Open Access gibt es interessante Neugründungen. Diese benötigen aber Zeit, um ihre Reputation aufzubauen.

Grundsätzlich gilt, dass jede Publikation nach ihrem wissenschaftlichen Inhalt, nicht aber nach der Zeitschrift, in der sie erschienen ist, $\mathrm{zu}$ bewerten ist.

Predatory Journals existieren auch in der Mathematik, stellen allerdings in der Regel kein ernsthaftes Problem dar. Es gibt allerdings Zeitschriften, welche sich von seriösen Journalen zu Predatory Journals entwickelt haben. In diesem Fall ist nicht automatisch von einer Veröffentlichung in einer solchen Zeitschrift auf ein wissenschaftliches Fehlverhalten zu schließen. Predatory Journals werden nicht in seriösen Datenbanken wie zbMATH oder MathSciNet dokumentiert.

Neben den klar erkennbaren Predatory Journals gibt es das Problem der Zeitschriften für „inner circles“, die vor allem dazu dienen, Zitationskartelle zu bedienen und Kennzahlen in die Höhe zu treiben. Naturgemäß ergibt sich hier eine Grauzone, deren Bewertung nicht immer einfach ist.

Es gehört zur Aufgabe der Betreuerinnen und Betreuer, den wissenschaftlichen Nachwuchs in Bezug auf die Auswahl geeigneter Journale zu beraten.

Es gibt (und gab) mehrere Versuche aus der Community heraus, ein Ranking von Journalen zu definieren. Allerdings 
sind diese Versuche nur mäßig erfolgreich. So wird das australische Ranking [29] derzeit nicht mehr aktualisiert, vgl. [19]. Die skandinavischen Fachgesellschaften haben ebenfalls ein Ranking erstellt, vgl. [30]. Hieraus ergibt sich allerdings höchstens eine grobe Einordnung, detaillierte Rückschlüsse sind nur sehr eingeschränkt möglich.

In jedem Fall ist festzustellen, dass es auch bei der Einordnung von Journalen starke gebietsspezifische Besonderheiten gibt.

Eventuelle Rankings müssten in regelmäßigen Abständen überprüft und aktualisiert werden. Insgesamt rät die DMV daher von der Verwendung der existierenden JournalRankings ab und hat sich deswegen auch bewusst dagegen entschieden, ein solches Ranking zu erstellen.

\section{Grundsätzliche Empfehlung}

Bibliometrische Daten werden heute in vielfältiger Weise verwendet, nicht zuletzt in Berufungsverfahren und bei der Bewertung von Anträgen. Die DMV appelliert an die Beteiligten, sich der Problematik bewusst zu sein, die eine unreflektierte Anwendung bibliometrischer Daten mit sich bringt. In diesem Zusammenhang verweist die DMV auf die San Francisco Declaration on Research Assessment [2].

Die Verwendung von bibliometrischen Daten zur Evaluation von Personen und Institutionen sollte stets mit einem Peer Review Verfahren verbunden werden. Dies gilt insbesondere für Entscheidungen, die unmittelbaren Einfluss auf die Karriere von Wissenschaftlerinnen und Wissenschaftlern haben.

\section{Glossar}

Altmetrics: Überwiegend webbasierte Metriken, die versuchen, die Reichweite von wissenschaftlichen Arbeiten z. B. durch Aggregation von Nutzungs- und Downloadzahlen oder Erwähnung in sozialen Netzwerken und Medien zu messen. Große Verlage haben hier durch aktive Öffentlichkeitsarbeit eine große Beeinflussungsmacht, ebenso gibt es naturgemäß einen Bias zugunsten öffentlichkeitswirksamer oder kontroverser Themen. Strukturell ist hier die Mathematik sowohl durch die geringe Größe als auch die erschwerte Zugänglichkeit benachteiligt. So hatte die AMS bei sich beispielsweise einige Jahre lang Altmetrics-Scores aktiviert, dies aber 2016 eingestellt, da praktisch keine Aussagekraft gegeben war. Ein drastisches Beispiel liefert die Arbeit [20] des Fields-Medaillenträgers Peter Scholze, die (lediglich) eine Altmetric-Score von 3 besitzt, vgl. [22]. Im Gegensatz dazu hat das NatureEditorial [21] zur MSC, das im wesentlichen auf drei Telefoninterviews basiert, einen Altmetric-Score von 145, siehe [23].

AMS: "American Mathematical Society", mit etwa 28000 Mitgliedern die weltweit größte mathematische Fachgesellschaft.

Autorendisambiguierung: Eindeutige Zuordnung von Autorensignaturen in Publikationen zu Personen, insbesondere Auflösung von Homonymen und Synonymen.
Directory of Open Access Journals (DOAJ): Das DOAJ ist ein internetbasiertes Verzeichnis von Open Access Journalen, welche einen Peer Review Prozess zur Qualitätskontrolle benutzen. Allerdings ist dieses Verzeichnis umstritten. Die EMS hat in ihrer Stellungnahme zum Plan S der EU [8] dazu festgestellt, dass aus ihrer Sicht DOAJ keine neutrale Instanz ist und eine Reihe von Zeitschriften aufführt, deren Praxis von der mathematischen Community als fragwürdig angesehen wird.

Eigenfaktor (EF): Bibliometrisches Maß, das wesentlich die Zentralität in Zitationsnetzwerken einbezieht, siehe [24]. Durch diesen Ansatz erfolgt eine gewichtete Zählung von Zitationen, vergleichbar dem Google PageRank. Inzwischen wird der EF auch in den ThomsonReuters Journal Citation Reports neben dem Impaktfaktor verwendet. Neben der Berechnung für Zeitschriften wird er auch als „Influence-Maß“ für Artikel oder Autoren verwendet. Strukturell benachteiligt er in hohem Maße Gebiete mit intrinsisch geringerer Publikations- und Zitationszahl, da diese automatisch als randwertig (nicht ,zentral“) in die Bewertung eingehen.

Google Scholar: Freie bibliographische Datenbank, deren Daten auf automatischer Extraktion und Nutzerbeiträgen beruhen. Eine Qualitätsprüfung findet nicht statt; Gebiets- oder Autorenzuordnungen sind oft heuristisch und leicht manipulierbar. Durch die automatisierte Herangehensweise ergibt sich jedoch ein Aktualitätsvorteil, insbesondere bei Preprints.

h-Index (Hirsch-Index): Ein 2005 von Jorge E. Hirsch vorgeschlagenes bibliometrisches Maß, [15]. Der $h$-Index ist die größte Zahl $h$, so dass ein Autor bzw. eine Autorin $h$ Arbeiten hat, die mindestens $h$-mal zitiert werden. Der $h$-Index eines Autors bzw. einer Autorin kann im Laufe der Zeit nicht kleiner werden. Es gibt eine Vielzahl von Modifikationen, z. B. nur die Berücksichtigung der Publikationen der letzten $x$ Jahre. Der $h_{m}$-Index berücksichtigt insbesondere die Anzahl der Verfasser einer Publikation. Der $h_{w}$-Index wiederum gewichtet die Zitationen nach ihrem Impakt. Darüber hinaus wurden eine Reihe von Modifikationen $(a$-index, $g$-Index, $k$-Index und diverse Kombinationen) vorgeschlagen, siehe [25]. In allen Fällen gilt, dass der $h$-Index stark von der Auswahl der Veröffentlichungen abhängt, aus denen er berechnet wird.

Impaktfaktor (IF): Historisch ältestes bibliometrisches Maß, zumeist berechnet von WoS als „Current Journal Impact Factor" als Quotient der Zitationszahl aller Artikel der letzten zwei Jahre einer Zeitschrift durch die Anzahl dieser Artikel berechnet. Eine Reihe von Untersuchungen belegt die vollkommen fehlende Aussagekraft im Bereich der Mathematik $[1,4,5,10]$.

Journal Citation Reports (JCR): Von Thompson Reuters jährlich herausgegebene bibliometrische Kennzahlen (Impaktfaktor, Eigenfaktor) von gebietsweise aufgeschlüsselten Zeitschriften.

Institutiondisambiguierung: Eindeutige Zuordnung von Autorensignaturen in Publikationen zu einer Institutionshierarchie. Auch die von Verlagen zur Verfügung 
gestellten Daten sind oft unvollständig (beispielsweise bei mehreren Autoren).

MathSciNet: Von der AMS herausgegebener Referatedienst für die Mathematik mit bibliographischer Datenbank und Zitationsinformationen [31]. Berechnet MCQ, einen 5-Jahres-Impatfaktor für Zeitschriften.

Predatory Journals: Zeitschriften mit einem als betrügerisch einzustufenden Geschäftsmodell von Verlagen, die sich als Open-Access-Publisher ausgeben, meist mit sehr professionell aufgemachten Websites auftreten und mittels offensiver und personalisierter EMail-Kampagnen versuchen, gegen Zahlung einer Gebühr Publikationen einzuwerben. Erfahrungsgemäß beziehen sich die E-Mails auf frühere Publikationen des Adressaten bzw. der Adressatin und es wird vorgetäuscht, im Fach etabliert $\mathrm{zu}$ sein sowie den wissenschaftlichen Publikationsstandards zu entsprechen. Die Helmholtz-Gemeinschaft bietet ausführliche Informationen $\mathrm{zu}$ diesem Thema an, vgl. [26]. Eine Liste mit Predatory Journals findet man unter [27].

ResearchGate: Eine kommerzielle Plattform, die sich als Social-Media-Service für Wissenschaftler versteht und neben anderen Funktionen auch eine eigene bibliometrische Kennziffer (RG Score) für Rankings verwendet. Die eigene Aktivität (und die des zugehörigen Forschungsnetzwerks) auf Researchgate beeinflusst diese Bewertung wesentlich, so dass dieser Score eher als Anreiz zur Nutzung der Plattform denn als Maß zur wissenschaftlichen Bewertung interpretiert werden kann.

Rankings: Verschiedene Institutionen veröffentlichen auf Grundlage primär bibliometrischer Maße regelmäBig Listen von Rankings von Institutionen (z. B. Times Higher Education, Academic Ranking of World Universities (ARWU) alias Shanghai ranking, CHE Hochschulranking etc.) oder Zeitschriften (Thompson Reuters, Scopus). Die Unsicherheiten bibliometrischer Maße werden hierbei z. B. durch Effekte der Institutionendisambiguierung verstärkt und führen schnell zu stark verzerrten Darstellungen.

Scopus: Kommerzielle Zitations- und Abstractdatenbank von Elsevier, die in großen Teilen ähnliche bibliometrische Maße wie das Web of Science anbietet.

Thomson Reuters Corp. Kommerzieller Anbieter von bibliometrischen Datenbanken und abgeleiteten Diensten, insbesondere Web of Science und Journal Citation Reports.

Web of Science (WoS): Online-Datenbank mit bibliographischen und Zitationsdaten (subskriptionsbasiert), früher Web of Knowledge/ISI Web of Knowledge, vertrieben von Thompson Reuters. Historisch wesentliche Triebkraft bei der Etablierung bibliometrischer Indikatoren, insbesondere des Impaktfaktors und abgeleiteter Rankings.

zbMATH: Von FIZ Karlsruhe, European Mathematical Society und Heidelberger Akademie herausgegebener Referatedienst für die Mathematik mit bibliographischer Datenbank und Zitationsinformationen [33].
Umfasst detaillierte Zitationsprofile, jedoch keine bibliometrischen Maße.

Zitationsfarmen: Artikel oder Journale, die auf Maximierung von Zitationen und bibliometrischen Kennzahlen hin optimiert werden, oft von zweifelhaftem wissenschaftlichem Wert (Beispiele: von El Naschie und/oder Ji-Huan He herausgegebene Zeitschriften indexiert im WoS; maschinelle Aufblähung durch Ausnutzung der Automatismen der Google Scholar Algorithmen).

\section{Quellen}

[1] Robert Adler, John Ewing, Peter Taylor: Zitaten-Statistiken. Mitt. Dtsch. Math.-Ver. 16, No. 3, 198-203 (2008).

[2] San Francisco Declaration on Research Assessment, https:// sfdora.org

[3] Ilka Agricola: Old and New on the Exceptional Group $G_{2}$. Notices Am. Math. Soc. 55 (8), 922-929 (2008)

[4] Douglas N. Arnold, Kristine K. Fowler: Nefarious numbers. Notices Am. Math. Soc. 58, No. 3, 434-437 (2011).

[5] Adam Bannister, Klaus Hulek, Olaf Teschke: Das Zitationsverhalten in mathematischen Arbeiten. Einige Anmerkungen. Mitt. Dtsch. Math.-Ver. 25, No. 4, 208-214 (2017)

[6] Adam Bannister, Olaf Teschke: An update on time lag in mathematical references, preprint relevance, and subject specifics. Eur. Math. Soc. Newsl. 106, 37-39 (2017) DOI: 10.4171/NEWS/106/15 [Zugriff am 7. März 2019]

[7] Thierry Bouche, Olaf Teschke, Krzyś Wojciechowski: Time lag in mathematical references. Eur. Math. Soc. Newsl. 86, 54-55 (2012) www.mathdoc.fr/publis/time_lag_in_mathematical_references. pdf [Zugriff am 7. März 2019]

[8] Feedback from the European Mathematical Society on the Guidance on the Implementation of Plan S, euro-math-soc. eu/system/files/news/EMS-PED-OA-PlanS.pdf [Zugriff am 27. April 2019]

[9] Rui Loja Fernandes: Evaluation of faculty at IST - a case study. Eur. Math. Soc. Newsl. 82, 13-17 (2011) faculty.math.illinois.edu/ $\sim$ ruiloja/ArtigoEMS.pdf [Zugriff am 7. März 2019]

[10] Antonia Ferrer-Sapena, Enrique Alfonso Sánchez-Pérez, Fernanda Peset, Luis-Millán González, Rafael Aleixandre-Benavent: The Lack of Stability of the Impact Factor of the Mathematical Journals. in: Proceedings of ISSI 2015 Istanbul: $15^{\text {th }}$ International Scientometrics and Informetrics Conference, Istanbul, Turkey, 29 June to 3 July, 2015 (2015)

[11] Sandra L. De Groote, Rebecca Raszewski: Coverage of Google Scholar, Scopus, and Web of Science: a case study of the hindex in nursing. Nurs Outlook, 6o(6), 391-40o (2012). DOI: 10.1016/j.outlook.2012.04.007 [Zugriff am 8. März 2019]

[12] Nikolaŭ Maksimovic Gyunter: Über die Elimination. St. Petersburg, Sborn. Inst. put. Soobšč. 83, 1-10 (1913)

[13] Nikolaĭ Maksimovic Gyunter: Über die kanonische Form der Systeme kanonischer homogener Gleichungen. Samml. des Inst. der Verkehrswege 82, 22 S. (Russisch) (1913)

[14] Nikolaĭ Maksimovic Gyunter: Über einige Zusammenhänge zwischen den homogenen Gleichungen. Samml. des Inst. der Verkehrwege 82 , 20 S. (1913)

[15] Jorge E. Hirsch: An index to quantify an individual's scientific research output. In: Proceedings of the National Academy of Sciences. Band 102, Nummer 46, S. 16569-16572 (2005) DOI: 10.1073/pnas.0507655102, arXiv:physics/0508025 [Zugriff am 8. März 2019]

[16] Kenji Iohara, Philippe Malbos: From analytical mechanical problems to rewriting theory through M. Janet. arXiv:1801.00053 [Zugriff am 8. März 2019] 
[17] Fabian Müller, Olaf Teschke: Will all mathematics be on the arXiv (soon)? Eur. Math. Soc. Newsl. 99, 55-57 (2016) In: www. ems-ph.org/journals/newsletter/pdf/2016-03-99.pdf [Zugriff am 8. März 2019]

[18] Walter Reichel: Über trilineare alternierende Formen in sechs und sieben Veränderlichen und die durch sie definierten geometrischen Gebilde. Dissertation Universität Greifswald, 1907

[19] Jill Rowbotham: End of an ERA: journal rankings dropped. The Australian. 30 May 2011. tinyurl.com/australian3omay2011 [Zugriff am 8. März 2019]

[20] Peter Scholze: Perfectoid Spaces. Publications mathématiques de l'IHÉS 116 (1), 245-313 (2012) DOI: 10.1007/s10240-012-0042-X [Zugriff am 8. März 2019]

[21] Editorial Maths classification is getting a revision. Nature, February 2018. DOI: 10.1038/d41586-018-0169o-2 [Zugriff am 8. März 2019]

[22] Digital Science \& Research Solutions Inc. Dimensions for [20]. tinyurl.com/altmetrics-scholze [Zugriff am 8. März 2019]

[23] Digital Science \& Research Solutions Inc. Dimensions for [21]. tinyurl.com/altmetrics-msc [Zugriff am 8. März 2019]

[24] The Eigenfactor Project. www.eigenfactor.org/about.php [Zugriff am 8. März 2019]
[25] Sammlung $h$-index and Variants. https://sci2s.ugr.es/hindex [Zugriff am 8. März 2019]

[26] Helmholtz Open Science: FAQs zum Thema "predatory publishing“. tinyurl.com/helmholtz-faqs [Zugriff am 8. März 2019]

[27] Stop Predatory Journals: List of Predatory Journals. predatoryjournals.com/journals/ [Zugriff am 8. März 2019]

[28] Journal Citation Reports: Journals in MATHEMATICS, Year 2017. tinyurl.com/JCRmath2017 [Zugriff am 7. März 2019]

[29] Journal Rankings for Australian Research Council Fields of Research: 01 - Mathematical Sciences. www.austms.org.au/Rankings/ AustMS_final_ranked.html [Zugriff am 8. März 2019]

[30] The Norwegian Register for Scientific Journals, Series and Publishers. tinyurl.com/nordiclist [Zugriff am 8. März 2019]

[31] Datenbank MathSciNet. mathscinet.ams.org/mathscinet/ [Zugriff am 8. März 2019]

[32] MathSciNet by the Numbers. mathscinet.ams.org/mathscinet/ help/byTheNumbers.html [Zugriff am 1. April 2019]

[33] Datenbank zbMATH. zbmath.org [Zugriff am 8. März 2019]

[34] zbMATH Facts \& Figures. https://zbmath.org/about/\#id_4 [Zugriff am 1. April 2019]

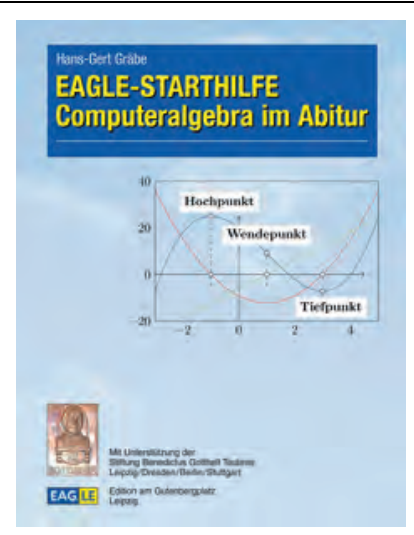

H.-G. Gräbe. EAGLE 104.

1. A. 2018. 978-3-95922-104-7

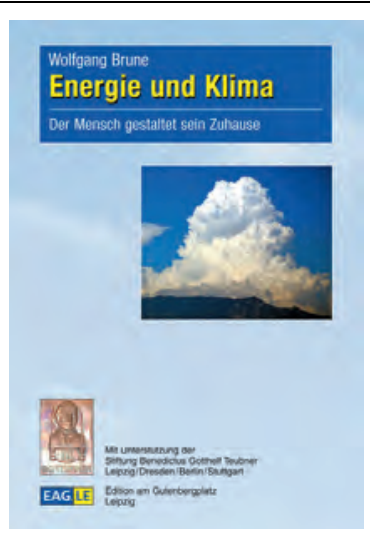

W. Brune. EAGLE 105. 1. A. 2019. 978-3-95922-105-4

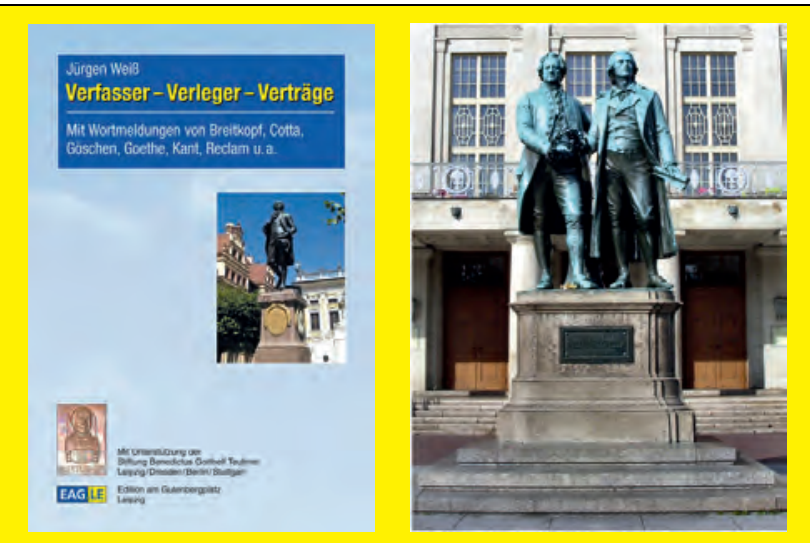

J. Weiß. Verfasser - Verleger - Verträge. Mit zahlreichen farbigen Abbildungen. EAGLE 106. HC. 1. A. 2019. 978-3-95922-106-1. Erscheint im Herbst 2019.

\section{E A L Leipzig, im Herbst 2019: Verfasser - Verleger - Verträge. Mit Wortmeldungen von Breitkopf, Cotta, Göschen, Goethe, Kant, Reclam u. a.: EA G.LE Brockhaus / Bürger / Fontane / Kleist / Lessing / Perthes / Schiller / Schlegel / Springer / Wieland. Literatur. Index. Mit zahlreichen Abbildungen. Unabhängiger Wissenschaftsverlag Edition am Gutenbergplatz Leipzig (EAGLE) / www.eagle-leipzig.de}

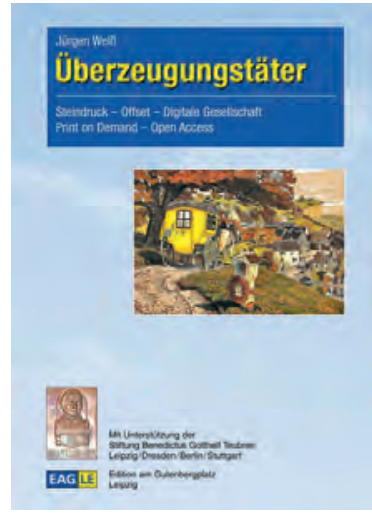

J. Weiß. EAGLE 100. HC. 1. A. 2017. 978-3-95922-100-9

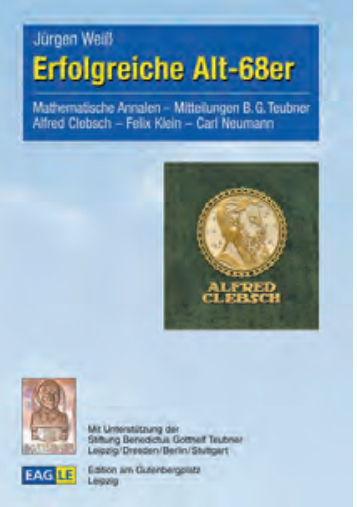

J. Weiß. EAGLE 101.

1. A. 2018. 978-3-95922-101-6

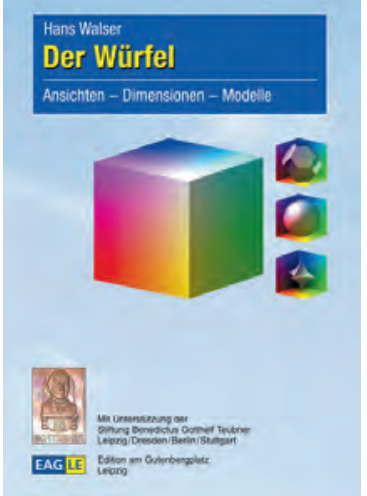

H. Walser. EAGLE 102. 1. A. 2018. 978-3-95922-102-3

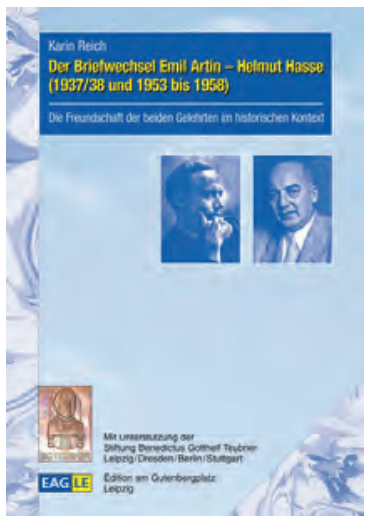

K. Reich. EAGLE 103.

1. A. 2018. 978-3-95922-103-0 